\title{
NOISE-LOOP MULTIPLE ACCESS FOR WIRELESS COMMUNICATIONS
}

\section{Mucchi, G. Chisci, E. Del Re}

\author{
University of Florence \\ Department of Information Engineering \\ via di Santa Marta 3, 50139, Firenze, Italy. \\ email: lorenzo.mucchi@unifi.it
}

\author{
L. S. Ronga \\ CNIT \\ University of Florence Unit \\ via di Santa Marta 3, 50139, Firenze, Italy.
}

\begin{abstract}
A fundamental problem for signal processing in wireless communications is the separation multiple signals/users from their mixture. The problem would be simpler if they are nonoverlapping in either time domain or frequency domain. Two signals may be overlapping in both time and frequency, but they still can be easily separated if they are uncorrelated (orthogonal), like in Code Division Multiple Access system (CDMA). It may be interesting to know that two signals may be overlapping in both the frequency and time domain and also are not orthogonal to each other, but they can still be separated if each autocorrelation function at the receiver is separable in the domain of time-lags. In this paper, we will show how the Noise-Loop (NL) modulation, originally thought to produce security at physical layer, can be used to effectively separate uncoordinated/unorthogonal users sharing the same time and frequency band. In order to show the potential of noise-loop multiple access (NLMA) scheme, the theoretical solution is derived as well as numerical semi-analytical simulations. It can be shown that the NLMA is able to distinguish the bit stream of the users. This feature together with the intrinsic security make the $\mathrm{NL}$ an interesting scheme for wireless technology.
\end{abstract}

Index Terms - multiple access, noise loop modulation, wireless networks.

\section{INTRODUCTION}

In a multiple access wireless system, many users share the same communication channel and hence they interfere with each other. However, these interferences can be easily taken care of by using the orthogonal structure of the multiple access systems. The interferences that are difficult to deal with are the ones that are not coordinated with the multiple access system they are using. Uncoordinated interference in unlicensed spectrum is the major road block for wireless communications. Typically, multiple access schemes separate users by forcing them to use a different time slot, different frequency interval or forcing them to be uncorrelated. Orthogonality can be provided by applying a code to each transmitting signal as in CDMA systems. Unfortunately, orthogonality of codes cannot be always assumed at the receiver, resulting in complex scheme to recover the orthogonality and synchronicity.

A key performance index for a multiple access system is the probability of error $\left(P_{e}\right)$ or the bit error rate (BER). It is known that $P_{e}$ is very sensitive to the total interference power at the access point (AP) demodulator [1]. In interference-limited systems, huge improvement in performance can be achieved by reducing the total interference at the receiver, when deciding on the symbol of each user, separately. Interference reduction techniques include, among others, sectorization, smart antennas, interference averaging, multiuser detection, and interference pre-cancellation [1]. The sectorization and the interference averaging methods have the objective of reduction but not elimination of the interferences. The pre-cancellation method requires channel state information (CSI) at the transmitter. The multiuser detection (MUD) method requires high computational effort as well as information about the distribution of the cochannel interferences which is usually not available.

In this paper we present a new multiple access scheme, called Noise-Loop Multiple Access (NLMA), which is able to separate the bit streams of uncoordinated users sharing the same time and frequency band. The noise-loop modulation was originally thought to produce security at physical layer on a peer-to-peer communication link [2], [3], [4]. We will show here how the NL modulation can be used as efficient and simple multiple access scheme. The proposed scheme keeps the peculiar characteristics of the single-user case in terms of complexity and latency of the secured link. This feature promotes it for potential application to simple radio devices, when an intrinsic security at radio layer is required (i.e. smart sensors for sensible data, body area networks, tactical radios).

The paper is structured as follows: in Section II a quick recall of Noise-Loop modulation is reported. Section III deals with the proposed multiple access extension of Noise-Loop. The main characteristics and performance are discussed in Section IV, while Section V provides concluding remarks. 


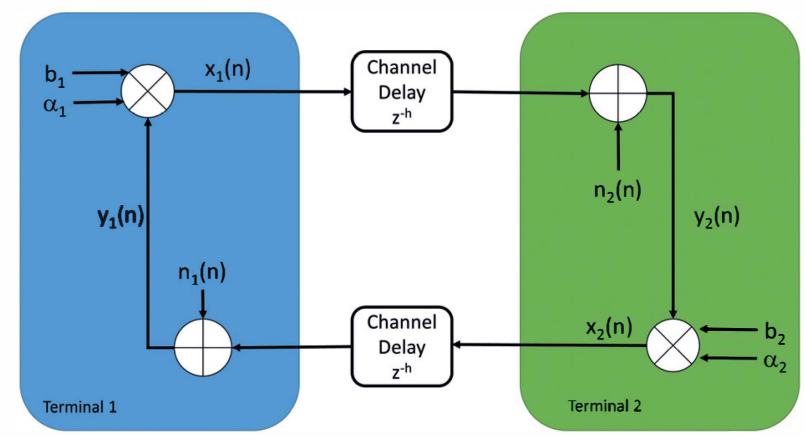

Fig. 1. The discrete-time model of the noise-loop system.

\section{RECALLING THE NOISE-LOOP MODULATION}

In order to recall the proposed technique [2], let us suppose two Terminals exchanging information, i.e., consider Terminal 1 (T1) and Terminal 2 (T2). Two different channels are considered: one for the link from Terminal 1 to Terminal 2 and one for the reverse link. The two channels are considered on different frequency bands and the thermal noise on one link is considered uncorrelated with the other. Each link is modeled as a conventional additive white Gaussian noise (AWGN) channel. The basic idea is that each Terminal takes what is coming at the receiver, modulates it with its information (plus noise) and sends it back to the other Terminal. This loop continues until enough energy is collected to demodulate correctly the bit. This loop interval is here called bit integration time. The receiver of each Terminal can easily demodulate the bit of the other Terminal by calculating the auto-correlation of the received signal at time lag equal to two-times the propagation delay between the two Terminals [2].

\section{II-A. Discrete-time Model of the Noise-Loop System}

The discrete-time model of the NL system is shown in Fig.1, where $b_{i} \in\{-1 ;+1\}$ is the binary antipodal information bit originating form Terminal $i=1,2, n_{i}(n)$ are the Gaussian, white, discrete-time random processes modeling the thermal noise at the $i$-th receiver (characterized by zero mean and variance $\left.\sigma_{n_{i}}^{2}\right), \alpha_{i}$ is the link gain $\left(0<\alpha_{i}<1\right)$ for the signal generated from Terminal $i, h_{i}$ is the propagation delay of the $i$-th channel, $x_{i}(n)$ is the transmitted signal from Terminal $i$ and $y_{i}(n)$ is the received signal available at the Terminal $i$. The signal from the inbound channel is modulated by the information and re-transmitted on the outbound channel. Let us focus, without loss of generality, on the reception of Terminal 1. In terms of time series, the

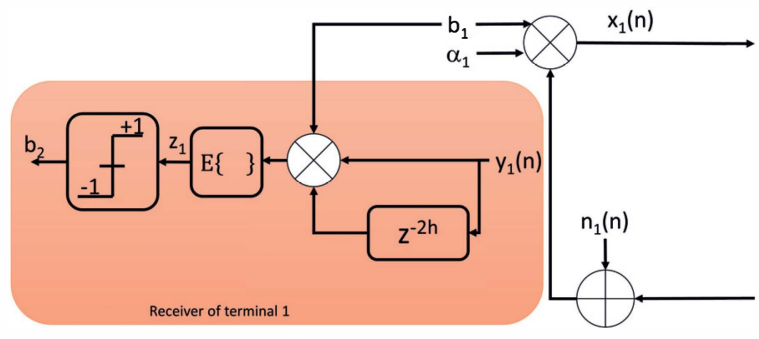

Fig. 2. The discrete-time model of the receiver for the Terminal 1 .

signal $y_{1}(n)$ can be written as [2]

$$
\begin{aligned}
y_{1}(n)=\sum_{j=0}^{+\infty}\left(K_{1} K_{2}\right)^{j} & n_{1}(n-2 j h)+ \\
& \sum_{j=0}^{+\infty}\left(K_{1} K_{2}\right)^{j} K_{2} n_{2}(n-(2 j+1) h)
\end{aligned}
$$

where $K_{i}=\alpha_{i} b_{i}$. The discrete-time received signal at Terminal 1 can be also written as a $2 h$-order autoregressive process

$$
y_{1}(n)=n_{1}(n)+K_{2} n_{2}(n-h)+K_{1} K_{2} y_{1}(n-2 h)
$$

The decision variable $b_{1} z_{1}$, which the Terminal 1 uses to demodulate the information symbol $b_{2}$, is simply the autocorrelation function of $y_{1}(n)$, evaluated at discrete-time lag $2 h$, multiplied by the information symbol $b_{1}$, i.e.,

$$
b_{1} z_{1}=b_{1} R_{y_{1}}(2 h)=b_{1}^{2} b_{2} \frac{\sigma_{n_{1}}^{2}+\alpha_{2}^{2} \sigma_{n_{2}}^{2}}{1-\alpha_{1}^{2} \alpha_{2}^{2}}=b_{2} \frac{\sigma_{n_{1}}^{2}+\alpha_{2}^{2} \sigma_{n_{2}}^{2}}{1-\alpha_{1}^{2} \alpha_{2}^{2}}
$$

The sign of $b_{1} z_{1}$ depends only on the sign of the symbol $b_{2}$, since the term $\frac{\sigma_{n_{1}}^{2}+\alpha_{2}^{2} \sigma_{n_{2}}^{2}}{1-\alpha_{1}^{2} \alpha_{2}^{2}}$ is always positive if the NL system is stable [2], i.e., $\alpha_{i} \in(0,1), i=1,2$.

The autocorrelation function

$$
R_{y_{1}}(2 h)=\mathrm{E}\left[y_{1}(n) y_{1}(n-2 h)\right]=b_{1} b_{2} \frac{\sigma_{n_{1}}^{2}+\alpha_{2}^{2} \sigma_{n_{2}}^{2}}{1-\alpha_{1}^{2} \alpha_{2}^{2}}
$$

can be derived from (2) by using the well known YuleWalker equations [2]. The reception is thus obtained by simply extracting the sign of the $2 h$-delayed autocorrelation term of the incoming signal, multiplied by the transmitted information bit. The discrete-time model of the receiver for Terminal 1 is shown in Fig.2.

\section{NOISE-LOOP MULTIPLE ACCESS}

The recalled Noise-Loop scheme has been originally introduced for ensuring security of a peer-to-peer radio link. In this paper, the goal is to show how the NL modulation can be exploited to provide a novel multiple access scheme. The 


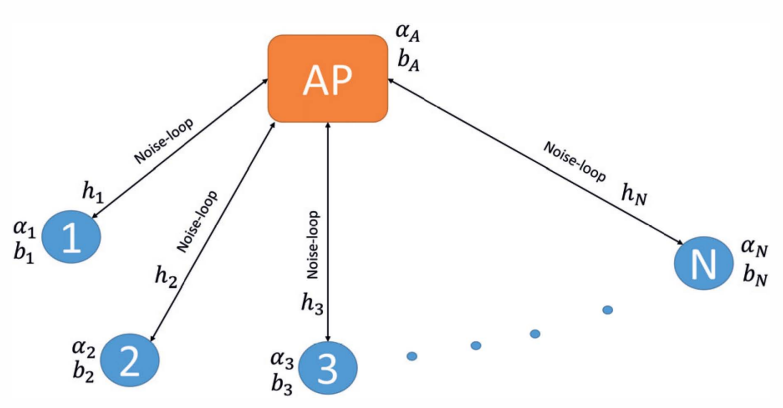

Fig. 3. The multiple access scheme.

system model is shown in Fig. 3. The system consists of an access point (AP) communicating with $N$ generic, randomly located, users. Each communication link is assumed to use the NL modulation. In particular, each user is supposed to have a gain $\alpha_{i} \in(0,1)$, a propagation discrete-time delay $h_{i}$ towards the AP and a BPSK information bit $b_{i} \in\{+1,-1\}$. Analogously to the P2P classical case (Eq. (1)), in the multiple users (MU) case the signal received by the $i$-th user $y_{i}(n)$ can be written as

$$
y_{i}(n)=n_{i}(n)+\underbrace{b_{A} \alpha_{A}}_{K_{A}} y_{A}\left(n-h_{i}\right)
$$

where $n_{i}(n)$ is the AWG noise at the $i$-th user's receiver, $\alpha_{A}$ is the transmission gain of the access point (AP), $b_{A}$ is the information bit transmitted by the $\mathrm{AP}$ and $h_{i}$ is the propagation delay between the $i$-th user's location and the AP. The signal received by the AP is

$$
y_{A}(n)=n_{A}(n)+\sum_{i=1}^{N} \underbrace{\alpha_{i} b_{i}}_{K_{i}} y_{i}\left(n-h_{i}\right)
$$

where $n_{A}(n)$ is the AWG noise at the AP's receiver, $\alpha_{i}$ is the transmission gain of the user $i$ and $b_{i}$ is the information bit transmitted by the user $i$. Let us focus on the signal $y_{A}(n)$ received by the AP which can be modeled as an autoregressive process, i.e.,

$$
y_{A}(n)=n_{A}(n)+\sum_{i=1}^{N} K_{i} n_{i}\left(n-h_{i}\right)+K_{A} \sum_{i=1}^{N} K_{i} y_{A}\left(n-2 h_{i}\right)
$$

Denoting with $e(n)$ the noise process

$$
e(n)=n_{A}(n)+\sum_{i=1}^{N} \alpha_{i} b_{i} n_{i}\left(n-h_{i}\right)
$$

we can rewrite Eq. (7) as

$$
y_{A}(n)=e(n)+\sum_{i=1}^{N} a_{i} y_{A}\left(n-2 h_{i}\right)
$$

where $a_{i} \triangleq K_{A} K_{i}=\alpha_{A} \alpha_{i} b_{A} b_{i}$. It is clear that the determination of the AR coefficient $a_{i}$ allows, therefore, to recover the unknown bit $b_{i}$, assuming the knowledge of $b_{A}$, as $b_{i}=b_{A} \operatorname{sgn}\left(a_{i}\right)$. To determine all AR coefficients, and hence all unknown bits, one can either solve the Yule-Walker equations

$$
\begin{aligned}
& \underbrace{\left[\begin{array}{c}
R_{y_{A}}\left(2 h_{1}\right) \\
R_{y_{A}}\left(2 h_{2}\right) \\
\vdots \\
R_{y_{A}}\left(2 h_{N}\right)
\end{array}\right]}_{\mathbf{r}}= \\
& \underbrace{\left[\begin{array}{ccc}
R_{y_{A}}(0) & \cdots & R_{y_{A}}\left(2 h_{N}-2 h_{1}\right) \\
\vdots & & \vdots \\
R_{y_{A}}\left(2 h_{1}-2 h_{N}\right) & \cdots & R_{y_{A}}(0)
\end{array}\right]}_{\mathbf{R}} \cdot \underbrace{\left[\begin{array}{c}
a_{1} \\
a_{2} \\
\vdots \\
a_{N}
\end{array}\right]}_{\mathbf{a}}
\end{aligned}
$$

with respect to a, or equivalently solve the linear least squares problem

$$
\min _{\mathbf{a}} \sum_{n=0}^{L}\left[y_{A}(n)-\varphi(n) \mathbf{a}\right]^{2}
$$

where $\varphi(n) \triangleq\left[y_{A}\left(n-2 h_{1}\right), y_{A}\left(n-2 h_{2}\right), \ldots, y_{A}\left(n-2 h_{N}\right)\right]$ and the summation extends over a bit integration interval of length $L \gg N$. As well known, the least-squares solution of (11) is given by

$$
\mathbf{a}_{L S}=\left(\sum_{n=0}^{L} \varphi(n)^{\top} \varphi(n)\right)^{-1} \sum_{n=0}^{L} \varphi(n)^{\top} y_{A}(n)
$$

The decision variable of AP for user $i$ is the sign of $z_{i}=$ $b_{A} a_{i}, a_{i}$ being estimated by the $i$ th element of vector $\mathbf{a}_{L S}$ in (12). This shows how the AP is able to demodulate the bit stream of each user without any code, frequency, or time dvision. The only assumptions are that all users experience different propagation delays, i.e. $h_{i} \neq h_{j}$ for any $i \neq j$, and that the noise loop is stable for any possible values of the bits $b_{A}, b_{1}, \ldots, b_{N} \in\{-1,+1\}$. The latter stability condition requires that the loop characteristic polynomial

$$
p(z)=1-\sum_{i=1}^{N} \beta_{i} \gamma_{i} z^{-2 h_{i}}
$$

be Schur stable, i.e. have all its roots strictly inside the unit circle, for any $\beta_{i}=b_{A} b_{i} \in\{-1,+1\}$ and fixed $\gamma_{i}=\alpha_{A} \alpha_{i}>0$. The following result provides necessary and sufficient conditions on the transmission gains $\alpha_{A}, \alpha_{1}, \ldots, \alpha_{N}$ for the stability of the multiple access noise loop of Fig. 3.

Theorem - The noise loop multiple access scheme of Fig. 3 is stable if and only if

$$
\sum_{i=1}^{N} \alpha_{i}<\frac{1}{\alpha_{A}}
$$


Proof. (Sufficiency) - Condition (14) implies $\sum_{i=1}^{N} \gamma_{i}<1$ which, in turn, implies

$$
\left|\sum_{i=1}^{N} \beta_{i} \gamma_{i} z^{-2 h_{i}}\right| \leq \sum_{i=1}^{N} \frac{\gamma_{i}}{|z|^{2 h_{i}}} \leq \sum_{i=1}^{N} \gamma_{i}<1 \Rightarrow \sum_{i=1}^{N} \beta_{i} \gamma_{i} z^{-2 h_{i}} \neq 1
$$

for all $z$ such that $|z| \geq 1$ and for all $\beta_{i} \in\{-1,+1\}$. Hence $p(z)$ in (13) cannot have roots in $|z| \geq 1$, i.e. is Schur stable, for any choice of $\beta_{1}, \ldots, \beta_{N}$ in $\{-1,+1\}$.

(Necessity) - If $p(z)$ is Schur stable for all $\beta_{i}$ then, taking $\beta_{i}=+1$ for all $i$, by the Schur stability criterion we have

$$
p(1)=1-\sum_{i=1}^{N} \gamma_{i}>0
$$

from which $\sum_{i=1}^{N} \gamma_{i}<1$ and, hence, (14) readily follows.

It can be shown (the proof is omitted here due to lack of space) that

$$
R_{y_{A}}\left(2 h_{i}\right)=a_{i} R_{y_{A}}(0)+o\left(\|a\|^{2}\right) \simeq a_{i} R_{y_{A}}(0)
$$

The sign of the autocorrelation function at lag $2 h_{i}$ is related to the sign of $a_{i}=b_{A} b_{i} \alpha_{A} \alpha_{i}$. Thus, the AP can demodulate the bit of user $i$ as $\hat{b}_{i}=\operatorname{sgn}\left[b_{A} \cdot R_{y_{A}}\left(2 h_{i}\right)\right]$ This approximate solution can be used in alternative to the exact one (12).

\section{III-A. Performance evaluation}

As depicted in Eq. (11), the decision variable for the AP can be derived by solving the LS problem. In particular, after a bit integration interval $L$, the vector of the signals received by the AP can be expressed as

$$
\mathbf{y}_{\mathbf{A}}=\Phi \cdot \mathbf{a}+\mathbf{e}
$$

where

$$
\begin{aligned}
\mathbf{y}_{\mathbf{A}} & =\left[y_{A}(n), \cdots, y_{A}(n+L)\right]^{T} \\
\mathbf{e} & =[e(n), \cdots, e(n+L)]^{T} \\
\Phi & =\left[\begin{array}{ccc}
y_{A}\left(n-2 h_{1}\right) & \cdots & y_{A}\left(n-2 h_{N}\right) \\
\vdots & \vdots & \vdots \\
y_{A}\left(n-2 h_{1}+L\right) & \cdots & y_{A}\left(n-2 h_{N}+L\right)
\end{array}\right]
\end{aligned}
$$

where ()$^{T}$ denotes the transpose operator.

The asymptotic LS estimated solution is known [8] to be normal distributed, thus

$$
\hat{\mathbf{a}}_{L S} \sim \mathscr{N}\left(\mathbf{a}, \sigma_{e}^{2}\left(\Phi^{H} \Phi\right)^{-1}\right)
$$

where ()$^{H}$ is the Hermitian operator. The performance of the AP's receiver can be defined as the probability of erroneous decision on the sign of the transmitted bits. Let us focus on the demodulation of the bit $b_{1}$ from user 1 , without loss of generality. The probability of error in this case is

$$
\begin{aligned}
& \operatorname{Pr}\left(\hat{a}_{1}<0 \mid b_{1}=1\right) P\left(b_{1}=1\right)+ \\
& +\operatorname{Pr}\left(\hat{a}_{1}>0 \mid b_{1}=-1\right) P\left(b_{1}=-1\right)=\operatorname{Pr}\left(\hat{a}_{1}<0 \mid b_{1}=1\right)= \\
& Q\left(\frac{\alpha_{1} \alpha_{A}}{\sqrt{2 \sigma_{e}^{2}\left[\left(\Phi^{H} \Phi\right)^{-1}\right]}}\right)
\end{aligned}
$$

The notation [ $]_{11}$ stands for extracting the first-row firstcolumn element of the matrix. Note that the decision variable for the AP is $z_{1}=b_{A} \hat{a}_{1}$ and bit $b_{A}$ is known by the AP's receiver. The variance $\sigma_{e}^{2}$ can be calculated looking at Eq. (8)

$$
\sigma_{e}^{2}=\sigma_{A}^{2}+\sum_{i=1}^{N} \alpha_{i}^{2} \sigma_{n_{i}}^{2}
$$

Fig. 4 shows the probability of error as a function of the loop gain $\alpha_{A}$ of the AP for the multiuser and single user detectors. Theoretical results and simulations are compared.

\section{RESULTS AND DISCUSSION}

Performance of the NLMA scheme based on (15) has been evaluated for variable number of users and bit integration time. The parameter $L$ is the length of the bit integration interval at the receiver and is expressed in samples, e.g., $L=$ 1024 means that the receiver integrates the decision variable over a window of 1024 samples.

Fig. 4 shows the probability of error of the NL solution with $N=\{4,8\}$ users and $L=512$. Both the multiuser detection (MUD) theoretical and the simulated results are shown. The theoretical LS MUD solution is obtained by (21). The GA MUD YW solution is obtained by solving (10) and then calculating the mean and variance of the solution for user 1 as if the decision variable was a Gaussian random variable, i.e., $P_{e}(x)=Q\left(\frac{\operatorname{Mean}[x]}{\operatorname{Var}[x]}\right)$. The MUD theoretical solutions are compared with the simulations. As seen, the theoretical solutions and simulations fit almost perfectly. This validates the simulation system implemented. Although the decision variable is not Gaussian, its statistics tend to be Gaussian, by the central limit theorem, as $L$ increases.

Fig. 5 shows performance of the NLMA scheme in terms of BER as a function of the AP transmission gain $\alpha_{A}$ for variable number of users $N=1 \rightarrow 16$ and bit integration interval $L=256 \rightarrow 4096$, but fixed ratio $L / N=256$. The main conclusions are: 1) theoretical results and simulations agree; 2) performance of the single-user and 16-user cases are very close, indicating that the NL is an efficient multiple access scheme, i.e., multiple access interference is rejected. Since performance of the NL scheme is invariant w.r.t. the noise power $\sigma_{e}^{2}$ [2], [3], [4], its value is not reported.

Fig. 6 shows the behaviour of BER versus $\alpha_{A}$ for variable number of users $N=1 \rightarrow 16$ but constant bit integration 


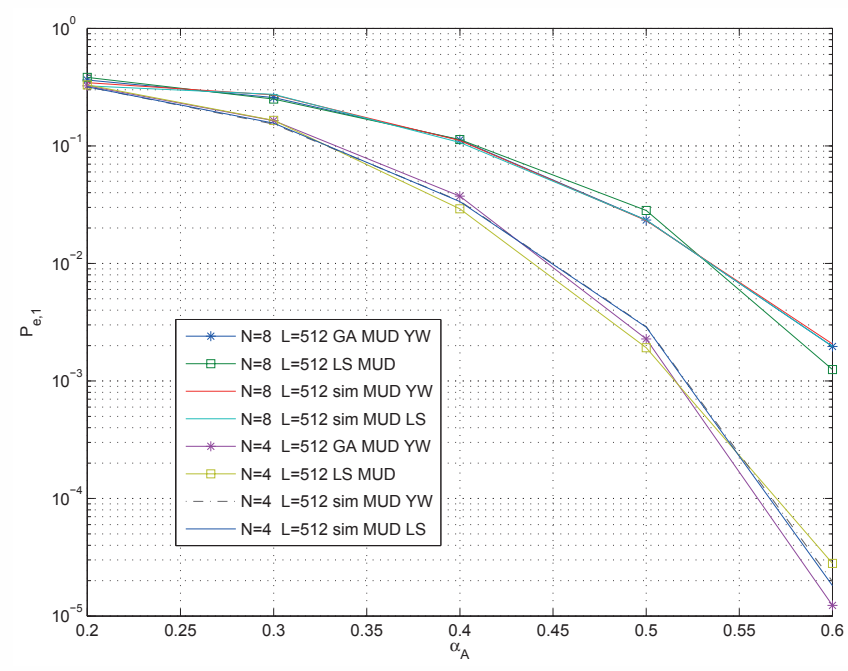

Fig. 4. Probability of error for the multiuser receiver with $N=\{4,8\}$ users and $L=512$. Theoretical solutions (LS, GA YW) are compared to simulations.

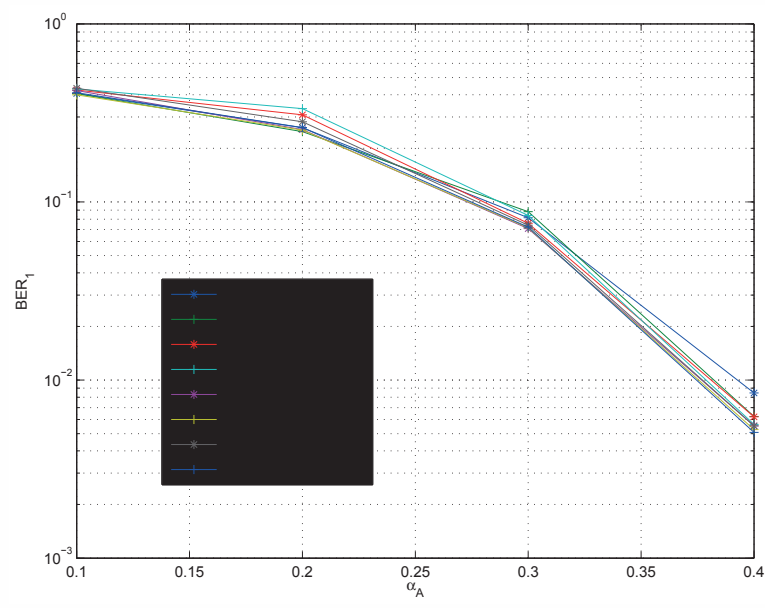

Fig. 5. BER of NLMA vs. $\alpha_{A}$ for $L / N=256$.

interval $L=1024$. As it can be seen, even in this case theory and simulations agree, but performance deteriorates as the number of users increases. This is because the ratio $L / N$ is not constant, implying that the loop gain of the generic $i$-th user decreases when $N$ increases (see (14)).

\section{CONCLUSIONS}

In this paper the Noise-Loop modulation scheme has been extended to the multi-user case. The theoretical derivations and computer simulations prove that the proposed scheme is indeed a new multi-user access scheme, where a constant bandwidth-per-user ratio results in a constant bit-error rate for the AWGN channel. Further investigations are ongoing to evaluate the impact on performance of more realistic

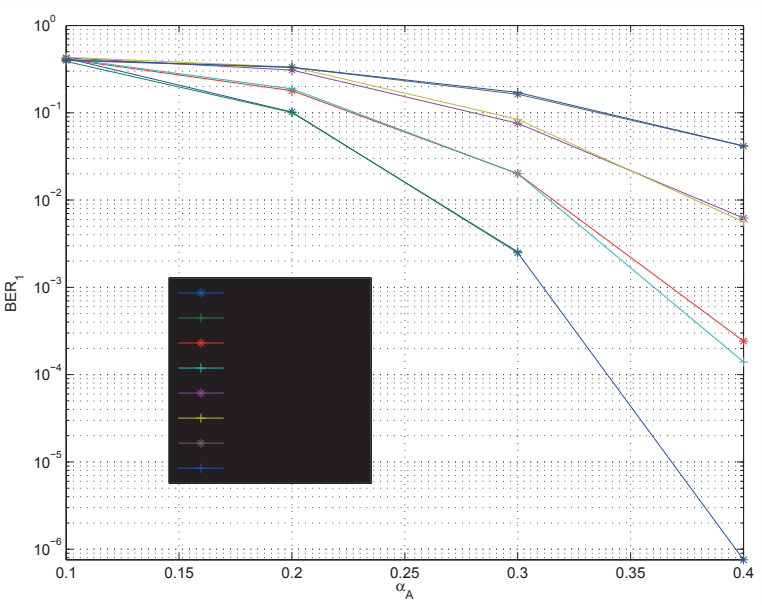

Fig. 6. BER of NLMA vs. $\alpha_{A}$ for $L=1024$ and $N=1,2,4,8$.

channel models. The authors feel also confident on the secrecy properties of the method inherited from the original Noise-Loop formulation, where a passive unwanted listener is unable to detect the information among friend users. This property, however, which is known to hold in the single-user case, must still be demonstrated for the multi-user case of this paper.

\section{REFERENCES}

[1] Andrea Goldsmith, Wireless Communications, Cambridge University Press, 2005.

[2] L. Mucchi. L. S. Ronga, L. Cipriani, A New Modulation for Intrinsically Secure Radio Channel in Wireless Systems, published in the special issue "Information Security and data protection in Future Generation Communication and Networking" of the Wireless Personal Communications (WPC) International Journal, by Springer; published online in 2008, printed in Volume 51, pag. 67-80, Number 1 / October, 2009; doi:10.1007/s11277-008-9609-8

[3] L. Mucchi, L. S. Ronga, E. Del Re, A novel approach for physical layer cryptography in wireless networks, Wireless Personal Communications Journal, Springer, 2010, doi:10.1007/s11277010-9950-6.

[4] L. Mucchi, L. S. Ronga, E. Del Re, Physical Layer Cryptography and Cognitive Networks, Wireless Personal Communications Journal, Springer, 2011, Volume 58, Issue 1, Pages: 95-109, doi:10.1007/s11277-011-0290-y.

[5] J. Proakis, Digital Communications, McGraw-Hill, 2001.

[6] A. Papoulis, Probability, Random Variables, and Stochastic Processes, McGraw-Hill, 1991.

[7] D.S.G. Pollock, A Handbook of Time-Series Analysis, Signal Processing and Dynamics, Academic Press, ISBN 0-12560990-6, 1999.

[8] Steven M. Kay, Fundamentals of Statistical Signal Processing: Estimation theory, Prentice-Hall PTR 1998. 